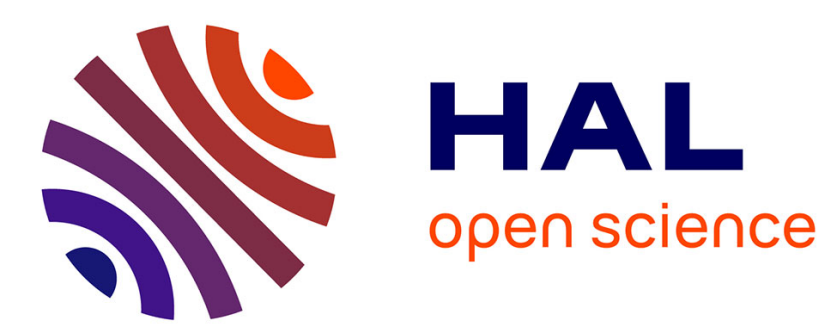

\title{
Simulation and Optimization of Stall Control for an Airfoil with a Synthetic Jet
}

Régis Duvigneau, Michel Visonneau

\section{To cite this version:}

Régis Duvigneau, Michel Visonneau. Simulation and Optimization of Stall Control for an Airfoil with a Synthetic Jet. Aerospace Science and Technology, 2006, 10 (4), pp.279-287. 10.1016/j.ast.2006.01.002 . hal-01730884

\section{HAL Id: hal-01730884 https://hal.inria.fr/hal-01730884}

Submitted on 14 Mar 2018

HAL is a multi-disciplinary open access archive for the deposit and dissemination of scientific research documents, whether they are published or not. The documents may come from teaching and research institutions in France or abroad, or from public or private research centers.
L'archive ouverte pluridisciplinaire HAL, est destinée au dépôt et à la diffusion de documents scientifiques de niveau recherche, publiés ou non, émanant des établissements d'enseignement et de recherche français ou étrangers, des laboratoires publics ou privés. 


\title{
Simulation and Optimization of Stall Control for an Airfoil with a Synthetic Jet
}

\author{
Régis Duvigneau and Michel Visonneau \\ Laboratoire de Mécanique des Fluides CNRS UMR 6598 \\ Ecole Centrale de Nantes \\ B.P. 92101, rue de la Noe FR-44321 Nantes, France \\ Michel.Visonneau@ec-nantes.fr
}

\begin{abstract}
This study concerns the simulation and optimization of stall control, using a synthetic jet. The flow is simulated by solving unsteady Reynolds-averaged Navier-Stokes equations with a near-wall low-Reynolds number turbulence closure. The flow around a NACA 0015 airfoil, including a synthetic jet located at $12 \%$ of the chord, is studied for a Reynolds number $R e=8.9610^{5}$ and for angles of attack from 12 to 24 degrees. The optimization of the control parameters (momentum coefficient, frequency, angle w.r.t. the wall) is intended, by coupling an automatic optimization algorithm with the flow solver. A significant improvement of the control efficiency is obtained (maximum lift increased of $34 \%$ and stall delayed from $19^{\circ}$ to $22^{\circ}$ w.r.t. the initial controlled flow). A Physical analysis of the flow is performed to characterize the optimal control process found.
\end{abstract}

Keywords : Flow control, Navier-Stokes, optimization, aerodynamic stall, synthetic jet

\section{Introduction}

Flow control using pulsated jets has been an active topic of research for many years. Indeed, flows can be modified by introducing jets with ad-hoc properties to obtain desired characteristics. At the present time, most applications concern aerodynamics problems. The aerodynamic properties of an airfoil, such as lift, drag or pitching moment, can be easily and quickly modified using flow control strategies, without modification of the angle of attack or flap deflection[13]. Most flow control techniques are based on jet suction and blowing. However, there are some difficulties to implement such devices into practical airfoils, since a large amount of power and room for air supply is required. Recently, an innovative actuator, called synthetic jet and based on high frequency oscillatory injection, was tested. It only requires electrical power 
and its efficiency was demonstrated experimentally by several authors[13].

Some numerical investigations concerning lift increase using synthetic jets were reported in the litterature, solving time-accurate Reynolds-Averaged Navier-Stokes equations. Wu et al.[14] investigated poststall lift enhancement for a NACA 0012 airfoil using a normal suction/blowing jet located at $2.5 \%$ of the chord and found that lift increase in the post-stall regime can be achieved, as was reported in experiments. The same approach was employed by Hassan et al.[8] for a slot at $13 \%$ of the chord, for different amplitudes and frequencies. It was reported that a high momentum coefficient was required to obtain a significant lift increase. Steady blowing as well as oscillatory jet actuations were simulated by Donovan et al.[3] and compared to experimental measurements. The same configuration was also studied by Ekaterinaris[6], who tested some different jet parameters.

All these numerical studies intend for describing the flow control process and for understanding the connection between the actuator parameters and the control efficiency. However, a systematic search "by hand" of the optimal control parameters is time consuming, since one time-accurate computation has to be performed for each attempt. Therefore, the automatic optimization of the parameters of a synthetic jet (momentum coefficient, frequency and angle w.r.t. the wall) is investigated in the present study, by coupling an optimization algorithm with a flow solver. The numerical methods used for the simulation of the flow are described in the first sections and applied to study the flow around a NACA 0015 airfoil including a synthetic jet. Then, the optimization procedure is detailed and applied to determine the optimal control parameters of the synthetic jet. Finally, a physical analysis of the flow is performed to understand why the optimal control found is efficient.

\section{Simulation of stall control}

\subsection{Numerical methods}

The numerical simulation of stall control is performed by the ISIS flow solver, developed by EMN (Equipe Modélisation Numérique i.e. CFD Department of the Fluid Mechanics Laboratory). The prediction of massively separated flows, such as flows encountered in stall control, is a difficult task. Although Large Eddy Simulation (LES) approaches may be more suitable for such flows, the present approach relies on solving Unsteady Reynolds-averaged Navier Stokes equations (URANSE). Indeed, the computational cost of LES approaches is still prohibitive for high Reynolds numbers, particularly in a design optimization framework.

Incompressible unsteady Reynolds-averaged Navier Stokes equations can be written as (using the gen- 
eralized form of Gauss' theorem):

$$
\begin{gathered}
\frac{\partial}{\partial t} \int_{V} \rho d V+\int_{S} \rho\left(\vec{U}-\vec{U}_{d}\right) \cdot \vec{n} d S=0 \\
\frac{\partial}{\partial t} \int_{V} \rho U_{i} d V+\int_{S} \rho U_{i}\left(\vec{U}-\vec{U}_{d}\right) \cdot \vec{n} d S=\int_{S}\left(\tau_{i j} I_{j}-p I_{i}\right) \cdot \vec{n} d S+\int_{V} \rho g_{i} d V
\end{gathered}
$$

where $V$ is the domain of interest, or control volume, bounded by the closed surface $S$ moving at the velocity $\vec{U}_{d}$ with a unit normal vector $\vec{n}$ directed outward. $\vec{U}$ and $p$ represent respectively the velocity and pressure fields. $\tau_{i j}$ and $g_{i}$ are the components of the viscous stress tensor and the gravity, whereas $I_{j}$ is a vector whose components vanished, except for the component $j$ which is equal to unity.

All the flow variables are stored at the geometric center of the arbitrary shaped cells. Volume and surface integrals are evaluated according to second-order accurate approximations. Numerical fluxes are reconstructed at the mesh faces, by using the values of the integrand extrapolated linearly from the neighboring cell centers. For the diffusive terms, a centered scheme is employed, whereas a combination of upwind and centered schemes are used for convective terms. It results from a local blending factor based on a continuous exponential scheme involving the signed Peclet number at the face [1]. Finally, the spatial discretization is second-order accurate.

A pressure equation is obtained in the spirit of the Rhie and Chow procedure. Momentum and pressure equations are solved in an segregated way like in the well-known SIMPLE coupling procedure.

The temporal discretization is based on a three-step scheme, yielding a second-order accuracy:

$$
\frac{\partial A}{\partial t} \approx e^{c} A^{c}+e^{p} A^{p}+e^{q} A^{q}
$$

where $c$ represents the current time step, $p$ and $q$ the past time steps. The coefficients $e^{c}, e^{p}$ and $e^{q}$ are chosen to ensure a second-order accuracy and depend on the steps length. All spatial terms appearing in equations (1a) and (1b) are discretized at the current time step, yielding an implicit scheme.

Several turbulence closures are included in the flow solver, ranging from linear eddy-viscosity based models to full second order closures [5]. For this study, the near-wall low-Reynolds number SST $k-$ $\omega$ model of Menter is chosen, since it behaves satisfactorily for separated flows over airfoils. Turbulent variables $k$ and $\omega$ are evaluated by solving transport equations which are discretized as the momentum equation. The capability of the flow solver to accurately predict turbulent flows has been demonstrated in the past (see for instance [4] for the validation of the flow around a three-element airfoil). 


\subsection{Computational results}

The flow control experiments of Gilarranz et al.[7] around a NACA 0015 airfoil for a Reynolds number $R e=8.9610^{5}$ are considered as a test case. However, the purpose of this study is not to reproduce these experiments, since they are characterized by three-dimensional and transitional effects. They are only considered as a starting point for the optimization problem.

To modelize the synthetic jet actuator, a suction/blowing type boundary condition is used. Thus, a prescribed velocity distribution is imposed at the jet boundary:

$$
\vec{U}=U_{j e t} \sin \left(2 \pi N_{\text {jet }} t\right) f(s) \vec{d}_{\text {jet }}
$$

where $\vec{d}_{j e t}$ is a vector of unit length representing the direction of the jet outlet. $\alpha_{j e t}$ is the angle between $\vec{d}_{j e t}$ and the wall (figure 1). $f(s)$ is the distribution of the velocity along the jet boundary. It is supposed to have a negligible influence on the flow, as shown by Donovan et al.[3]. Therefore, a "top hat" distribution is adopted, corresponding to $f(s)=1$. Concerning the turbulent variables, zero Neumann boundary conditions are imposed on the jet boundary for $k$ and $\omega$.

In the experiments of Gilarranz et al.[7], the actuation is performed by a synthetic jet located at $S_{j e t}=$ $12 \%$ of the chord $L$ from the leading edge, with a slot width $h=0.53 \%$ of the chord (figure 2). Among the parameters values tested experimentally, a non-dimensional frequency $N_{j e t}=N_{\text {exp }} L / U_{\infty}=1.29$ and a non-dimensional velocity amplitude $U_{\text {jet }}=U_{\text {exp }} / U_{\infty}=1.37$ are retained because of the satisfactory results obtained (with $U_{\infty}$ the free stream velocity, $N_{\exp }$ the frequency of the jet and $U_{\exp }$ the velocity amplitude of the jet). The jet outlet is almost tangential to the wall. Then, we impose a small angle $\alpha_{j e t}=$ $10^{\circ}$ for the conputations. These values correspond to a momentum coefficient $C_{\mu}=h U_{\text {exp }}^{2} / L U_{\infty}^{2}=$ 0.0099 .

An unstructured grid including 84577 cells is used for the calculations. This grid is composed of a fine mesh with quadrangular cells close to the wall and in the near wake, in order to get an accurate description of the vortex shedding, and a triangular mesh around to reduce the grid size. The distance between the first node and the wall corresponds to $y^{+}=0.3$. The number of nodes on the suction side of the airfoil is 364 and a refined area is located at the jet slot, which is described by 45 faces (figure 2). All computations are performed on the same mesh.

A non-dimensional time step $\Delta t=5 \cdot 10^{-3}$ is chosen in order that each flow cycle be described by a sufficient number of time steps. Time-accurate calculations are initialized with uniform flow fields and are performed until the non-dimensional time $t=t_{\exp } U_{\infty} / L=70$ (with $t_{\text {exp }}$ the physical time), for which a periodic flow is observed.

To analyse the physical phenomena involved in the control process, we consider first the flow around 
a NACA 0015 airfoil without actuation (referred as "baseline airfoil"), and then the flow around an airfoil including a synthetic jet with the control parameters described above (referred as "controlled airfoil with experimental parameters"). Figures 3 and 4 show the evolution of the time-averaged lift and drag coefficients w.r.t. the angle of attack. On these figures, error bars represent the variations of the intantaneous aerodynamic coefficients along time. For the baseline airfoil, the flow is rather steady until $20^{\circ}$. Stall occurs at $16^{\circ}$, caused by a growing recirculation zone which appears for $14^{\circ}$ at the trailing edge. In the post-stall regime, after $22^{\circ}$, the flow is detached on the whole suction side of the airfoil and a counter-rotative vortices shedding occurs, yielding a severe drag increase. For the controlled airfoil with experimental parameters, the flow is always unsteady, due to the presence of the oscillatory jet. It generates some small vortices which are convected downstream along the suction side of the airfoil. The actuation does not modify the time-averaged aerodynamic coefficients until $14^{\circ}$, but it maintains the lift increase until $18^{\circ}$. At $20^{\circ}$ and $22^{\circ}$, the lift is still higher than that of the baseline airfoil, although decreasing, and the drag increase is restrained. However, at $24^{\circ}$ the actuation has no more influence on the flow, which is characterized by the same counter-rotative vortices shedding as the flow around the baseline airfoil. It yields a strong increase of the drag and the variation of the efforts along time is growing. Finally, the comparison of the drag polar for the baseline airfoil and the controlled airfoil with experimental parameters (figure 5) shows that the benefit of the control is substantial, but limited from $16^{\circ}$ and $20^{\circ}$.

\section{Optimization of stall control}

\subsection{Methodology}

Design optimization consists in maximizing an objective function $F$ which depends on the design variables $D$ and the flow variables $Q(D)$. The governing equations of the flow $R(D, Q(D))=0$ are considered as constraints which must be satisfied at each step of the design procedure. Some bound constraints must be added to the problem in order to find a realistic solution. Thus, the variation domain of the design variables is usually closed. From a mathematical point of view, the problem may be expressed as:

$$
\begin{array}{ll}
\text { Maximize } & F(D, Q(D)) \\
\text { Constrained to } & R(D, Q(D))=0 \\
& L_{i} \leq D \leq L_{s}
\end{array}
$$

In the present work, the objective function is the time-averaged lift coefficient $C_{l}(D, Q(D))$ of the airfoil. It is evaluated after any transient effects have vanished, by integrating the instantaneous lift coefficient 
during a sufficient long time to take into account several flow cycles:

$$
F(D, Q(D))=\frac{1}{t_{2}-t_{1}} \int_{t_{1}}^{t_{2}} C_{l}(D, Q(D)) d t
$$

In practice, the above integration is performed during about 1000 time steps. Some numerical experiments have shown that this interval of integration is large enough in order that the time dependency be negligible.

The design variables $D$ are the control parameters of the synthetic jet: the velocity amplitude $U_{j e t}$, the jet frequency $N_{j e t}$ and the direction of the outlet $\alpha_{j e t}$.

Therefore, the design procedure consists in several unsteady flow simulations for a synthetic jet with different control parameters, whose values are modified by an optimization algorithm. Thus, the design procedure is described by :

(1) Initialization of the design variables $D$

(2) Unsteady simulation of the flow $Q(D)$

(3) Evaluation of the time-averaged lift $F(D, Q(D))$

(4) Update of $D$ by the optimization algorithm

(5) Goto step (2)

until the convergence of the design variables is achieved. This design cycle is performed for each angle of attack, in order to observe its influence on the optimal control parameters found.

In optimization, the use of gradient-based methods is usually motivated by their efficiency, since they can reach a maximum of the objective function in a number of evaluations lower than zero-order methods. However, some difficulties arise when they are faced with complicated realistic problems. The evaluation of the derivatives of the objective function for a sophisticated simulation process is rather problematic. Their evaluation is usually based on an adjoint formulation, which relies on the differentiation of the flow solver $[11,9]$. This task is tedious when high-order discretization schemes on unstructured grids are used, or complex turbulence models are employed. This approach often needs an a-priori simplification of the problem, neglecting turbulence sensitivities for instance, or using first-order discretization schemes, which provides an approximated gradient. It has been shown by Anderson and Nielsen [11] that these simplifications often lead to erroneous gradient values. Moreover, these algorithms are very sensitive to the noisy errors arising from the evaluation of the objective function and generating irregularities and spurious local maxima. These sources of errors were studied by Madsen [10], who underlined the high-frequency errors introduced by the use of high-order discretization schemes and low converged solutions. Thus, this approach is still limited to moderately complicated problems and its use for massively separated flows is in 
doubt.

To overcome these limitations, we choose to employ a derivative-free algorithm, which is easier to implement in a complex numerical framework. It can be easily associated with a sophisticated flow solver, since the solver is considered as a black-box and is not modified when included in the design procedure. Furthermore, this approach is less sensitive to the noise, because no information about the derivatives is needed to predict the optimization path. The number of evaluations required is higher than that necessited by gradient-based methods, but it remains reasonable, as soon as the number of design variables is low, which is the case in the present study.

The optimization method used to lead the search is based on the multi-directional search algorithm developped by Torczon[2]. This algorithm is inspired from the Nelder-Mead simplex method, used by the authors in the past for shape optimization purpose[4, 5].

This algorithm consists in moving a simplex of $n+1$ vertices in $\mathbb{R}^{n}$ (a triangle in $\mathbb{R}^{2}$, a tetrahedron in $\mathbb{R}^{3}$, etc), for a problem of $n$ design parameters, each vertex representing a distinct design. The moves are performed reflecting the whole simplex with respect to the best vertex, until a maximum is reached. The simplex can eventually be expanded or contracted to adapt itself to the local topology of the objective function.

\subsection{Computational results}

Between 15 and 20 optimization steps are required to reach the stopping criterion of the algorithm, for which design variables have converged to their optimal values. It corresponds to about 45 to 60 unsteady simulations for each angle of attack. Since it represents a large computational cost, a multi-block domain partitioning approach is used. Actually, each optimization exercise takes about 100 hours of elapsed time using 16 processors.

As can be seen in figure 3, the optimizer fails to significantly increase the lift as long as the flow is fully attached. Nevertheless, between $14^{\circ}$ and $18^{\circ}$, the control with optimal parameters provides a more efficient actuation than that with the experimental parameters, since the slope of the lift coefficient w.r.t. the angle of attack is slightly increased. Moreover, the actuation is efficient for higher angles of attack, since the stall is delayed to $22^{\circ}$, which corresponds to an increase of $34 \%$ of the maximum lift coefficient w.r.t. the airfoil with experimental parameters. These results clearly show that the choice of the control parameters is critical to obtain a large stall delay. However, the optimal control found is characterized by a larger variation of the efforts along time for all angles of attack. The drag is similar for the two sets of parameters from $12^{\circ}$ to $20^{\circ}$ (figure 4). At $22^{\circ}$, the drag obtained for optimal parameters is characterized by a severe increase. As explained below, it is due to the use of a very different actuation frequency. At 
$24^{\circ}$, the drag using optimal parameters is far lower than that using experimental parameters. Indeed, it was found that the experimental control has no more influence on the flow for this incidence, contrary to the optimal control. Finally, the comparison of the drag polar (figure 5) puts in light the benefit obtained thanks to the optimization procedure.

The optimal design parameters found are shown on table 1 . Except for the incidence $12^{\circ}$, for which no significant improvement is observed, some tendancies can be drawn. First, the amplitude of the jet velocity $U_{j e t}$ is increased to a mean value of $\overline{U_{j e t}^{o p t}}=1.72$. The increase of the control efficiency and the enlargement of the lift variation as the velocity amplitude grows was reported by some numerical studies $[3,6,12]$ as well as experimental observations[13]. Then, the angle between the jet outlet and the wall is increased to a a mean value of about $\overline{\alpha_{j e t}^{o p t}}=25^{\circ}$. This tendency is in agreement with the computations of Ekaterinaris[6] for trailing edge control and Donovan et al.[3] for leading edge control. The most interesting parameter is the frequency. Indeed, except for the incidence $12^{\circ}$ for which no significant improvement is observed, most optimal frequencies found are very close to each other, with a mean value of $\overline{N_{j e t}^{o p t}}=0.85$. This decrease of the frequency may also be responsible for the increase of the lift variation, as reported in the litterature[6,13]. Only one optimal value, at $22^{\circ}$, significantly differs, since the optimal frequency found is $N_{\text {jet }}^{\text {opt } 22}=0.25$. For this very low actuation frequency, the flow is characterized by a large vortex shedding, which is responsible for the severe drag increase, although the lift is maintained.

A physical analysis of the flow is now performed to understand how these optimal parameters can generate an efficient control process. The characteristics of the flow for an angle of attack of $20^{\circ}$ are compared for experimental and optimal control parameters. This analysis is not performed at the incidence $22^{\circ}$, which corresponds to the maximum lift, since the very low optimal frequency found at this incidence can be considered as doubtfull. The analysis is focused on the comparison of the lift history, the pressure coefficient at the wall, the streamlines and the velocity profiles at different stations for experimental and optimal control parameters.

The lift history (figure 6) shows a tendency to move away from a simple sinusoidal curve. The variation of the lift is larger, but the time-averaged lift remains higher than that with the initial parameters, at all times. To understand the reasons of this higher lift, consider the streamlines on figure 7 . It provides a snapshot of the flow just at the end of suction, which corresponds to the phase $\Phi=2 \pi N_{j e t} t=0$. As can be seen, three vortices are convected along the suction side of the airfoil for experimental parameters, whereas only two vortices are observed for optimal parameters. This characteristic is due to the fact that the actuation frequency is decreased. Therefore, the flow can be considered as more attached in the second case. It provides a first explaination of the higher lift observed for the optimal parameters. The presence of the recirculation zones is also noticed on figure 8, which represents the pressure coefficient at the wall, computed for experimetal parameters and optimal parameters. It yields pressure oscillations on the suction 
side of the airfoil. However, this figure shows that the main reason of the higher lift is due to the presence of a far higher suction peak for the optimal parameters $(+31 \%)$. The velocity profiles at different stations are computed and compared for experimental parameters and optimal parameters in figure 9 . The $2 \%$ chord station corresponds to the suction peak location, whereas the $10 \%$ chord station is located just upstream the slot. As can be seen, optimal control parameters yield higher velocities in the boundary layer (about $+10 \%$ ) whatever the station, which maximizes suction effects. This velocity increase is observed in the boundary layer as well as in the outer zone, and finally vanishes as one moves away from the airfoil. In conclusion, these comparisons show that the efficiency of the control is mainly related to the capability of the actuation to accelerate the flow in the vicinity of the leading edge and to minimize the detachment area on the suction side of the airfoil.

To have a better description of the flow dynamics, streamlines, velocity profiles and pressure coefficients at different times in a cycle are represented in figures 10, 11 and 12 for the optimal parameters. The phase $\Phi=0$ corresponds to the end of suction with a zero mass flow, $\Phi=\pi / 2$ to the maximum blowing, $\Phi=\pi$ to the end of blowing with a zero mass flow and $\Phi=3 \pi / 2$ to the maximum suction. The streamlines history is correlated to the lift history during a cycle (figure 10). As can be seen, a counter-rotative vortices shedding occurs at the beginning of the blowing stage, which corresponds to the lowest lift times. Then, the lift increases from the maximum blowing time to the maximum suction time. New vortices are generated on the suction side at these times. Then, these vortices grow and the lift decreases as the suction is reduced. The pressure coefficient history (figure 11) shows that the suction peak intensity is almost constant during the whole cycle, ensuring a high time-averaged lift. Then, lift variations are due to the convection of vortices along the suction side of the airfoil. The velocity profiles at different stations shown in figure 12, confirm that the oscillatoring flow actuation generates variations of the velocity profiles close to the slot. Especially, the velocity upstream of the slot is increased during the suction stage. However, the influence on the velocity profiles far upstream is almost independent of the time, as the suction peak intensity.

\section{Conclusion}

The simulation of aerodynamic stall control using a synthetic jet actuator is performed, by solving unsteady Reynolds-averaged Navier-Stokes equations, for a NACA 0015 airfoil and a Reynolds number $R e=8.9610^{5}$. The flow solver is then coupled with an automatic optimization procedure, which relies on a derivative-free multi-directional search algorithm. For each angle of attack, the velocity amplitude, the frequency and the angle w.r.t. the wall are optimized to increase the time-averaged lift. Experimental parameters are chosen as starting parameters for the optimization.

It is found that the efficiency of the control is significantly improved when optimal parameters are 
chosen. The maximum lift is increased by $+34 \%$ and the stall delayed from $19^{\circ}$ to $22^{\circ}$, w.r.t. the experimental parameters. The optimal parameters are characterized by an increase of the velocity amplitude and a decrease of the actuation frequency.

The physical analysis of the flow shows that the efficiency of the control is mainly related to the capability of the actuation to accelerate the flow in the vicinity of the leading edge and to minimize the detachment area on the suction side of the airfoil.

\section{Acknowledgements}

The authors gratefully acknowledge the scientific committee of CINES (project dmn2050) and IDRIS (project 1308) for the attribution of CPU time.

\section{References}

[1] I. Demirdžić and S. Muzaferija. Numerical method for coupled fluid fbw, heat transfert and stress analysis using unstructured moving meshes with cells of arbitrary topology. Comput. meth. Appl. Mech. Eng., 125:235-255, 1995.

[2] J.E. Dennis and V. Torczon. Direct search methods on parallel machines. SIAM Journal of Optimization, 1(4):448474, 1991.

[3] J.F. Donovan, L.D. Kral, and A.W. Cary. Active fbw control applied to an airfoil. AIAA Paper 98-0210, January 1998.

[4] R. Duvigneau and M. Visonneau. Shape optimization for incompressible and turbulent fbws using the simplex method. AIAA Paper 2001-2533, June 2001.

[5] R. Duvigneau, M. Visonneau, and G.B. Deng. On the role played by turbulence closures for hull shape optimization at model and full scale. Journal of Marine Science and Technology, 8(1), June 2003.

[6] J.A. Ekaterinaris. Active fbw control of wing separated fbw. ASME FEDSM'03 Joint Fluids Engineering Conference, Honolulu, Hawai, USA, July 6-10, 2003.

[7] J.L. Gilarranz, L.W. Traub, and O.K. Rediniotis. Characterization of a compact, high power synthetic jet actuator for fbw separation control. AIAA Paper 2002-0127, September 2002.

[8] A.A. Hassan, F.K. Straub, and Charles B.D. Effects of surface blowing/suction on the aerodynamics of helicopter rotor blade-vortex interactions - a numerical simulation. Journal of American Helicopter Society, 42:182-194, April 1997.

[9] A. Jameson, L. Martinelli, and N. A. Pierce. Optimum aerodynamic design using the Navier-Stokes equation. Theorical and Computational Fluid Dynamics, 10:213-237, 1998.

[10] J.I. Madsen. Response surface techniques for diffuser shape optimization. AIAA Journal, 38(9):1512-1518, 2000. 
[11] E. Nielsen and W. K. Anderson. Aerodynamic design optimization on unstructured meshes using the NavierStockes equations. AIAA Journal, 37(11):1411-1419, 1999.

[12] S.S. Ravindran. Active control of fbw separation over an airfoil. Technical Report TM-1999-209838, NASA, 1999.

[13] A. Seifert, A. Darabi, and I. Wygnanski. Delay of airfoil stall by periodic excitation. AIAA Journal, 33(4):691707, July 1996.

[14] J.M. Wu, X.Y. Lu, A.G. Denney, M. Fan, and J.Z. Wu. Post-stall lift enhancement on an airfoil by local unsteady control, part i. lift, drag and pressure characteristics. AIAA paper 97-2063, 1997. 


\section{List of Figures}

Fig. 1 Problem description

Fig. 2 Mesh close to the jet

Fig. 3 Lift coef. with respect to the angle of attack

Fig. 4 Drag coef. with respect to the angle of attack

Fig. 5 Drag polar

Fig. 6 Lift coef. history at $20^{\circ}$

Fig. 7 Comparison of the streamlines for $\Phi=0$

Fig. 8 Comparison of the pressure coefficients for $\Phi=0$, computed for experimental and optimal parameters

Fig. 9 Comparison of the velocity profiles for $\Phi=0$, computed for experimental and optimal parameters

Fig. 10Streamlines for different times

Fig. 11Pressure coefficients for different times

Fig. 12Velocity profiles for optimal parameters 


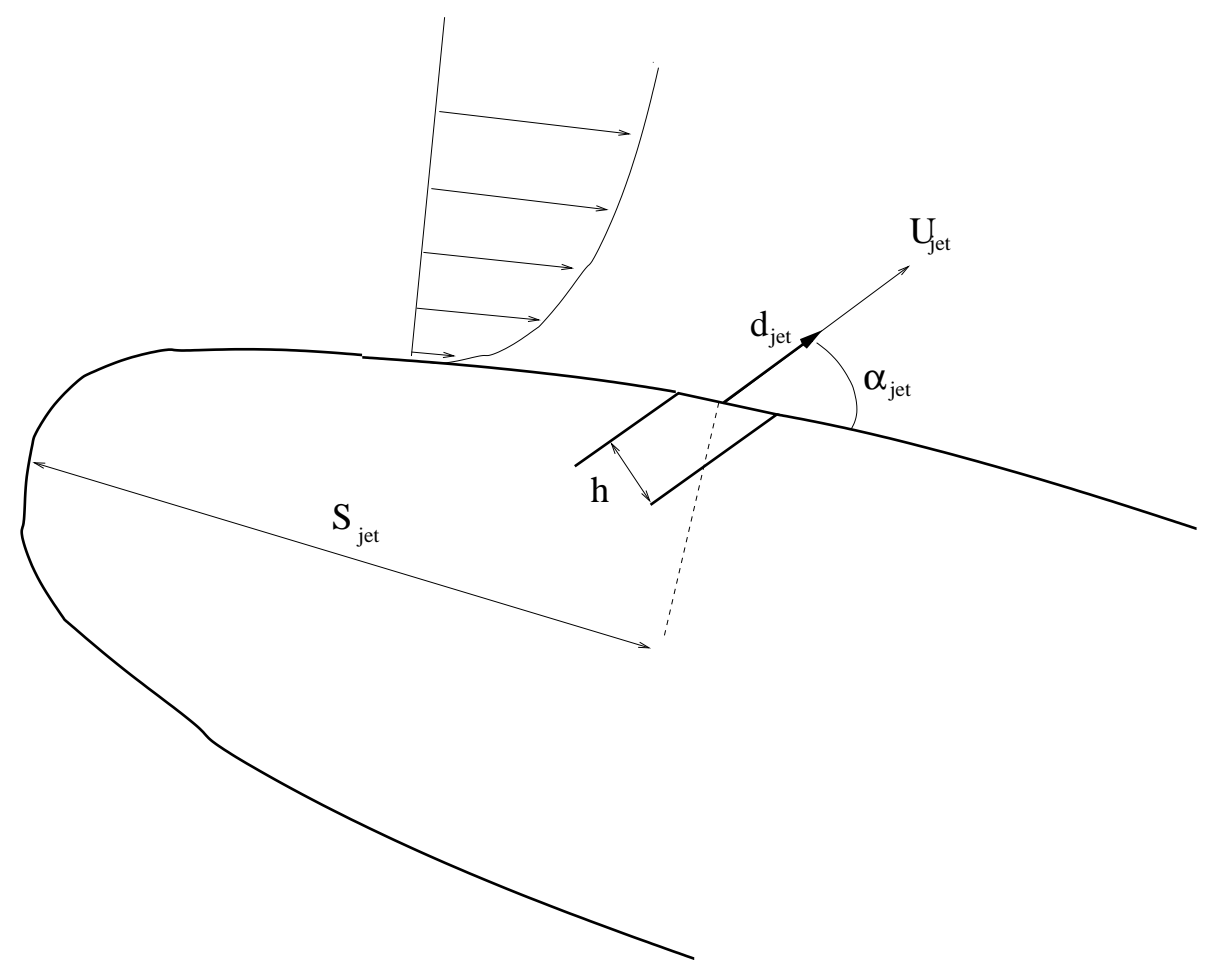

Figure 1: Problem description 


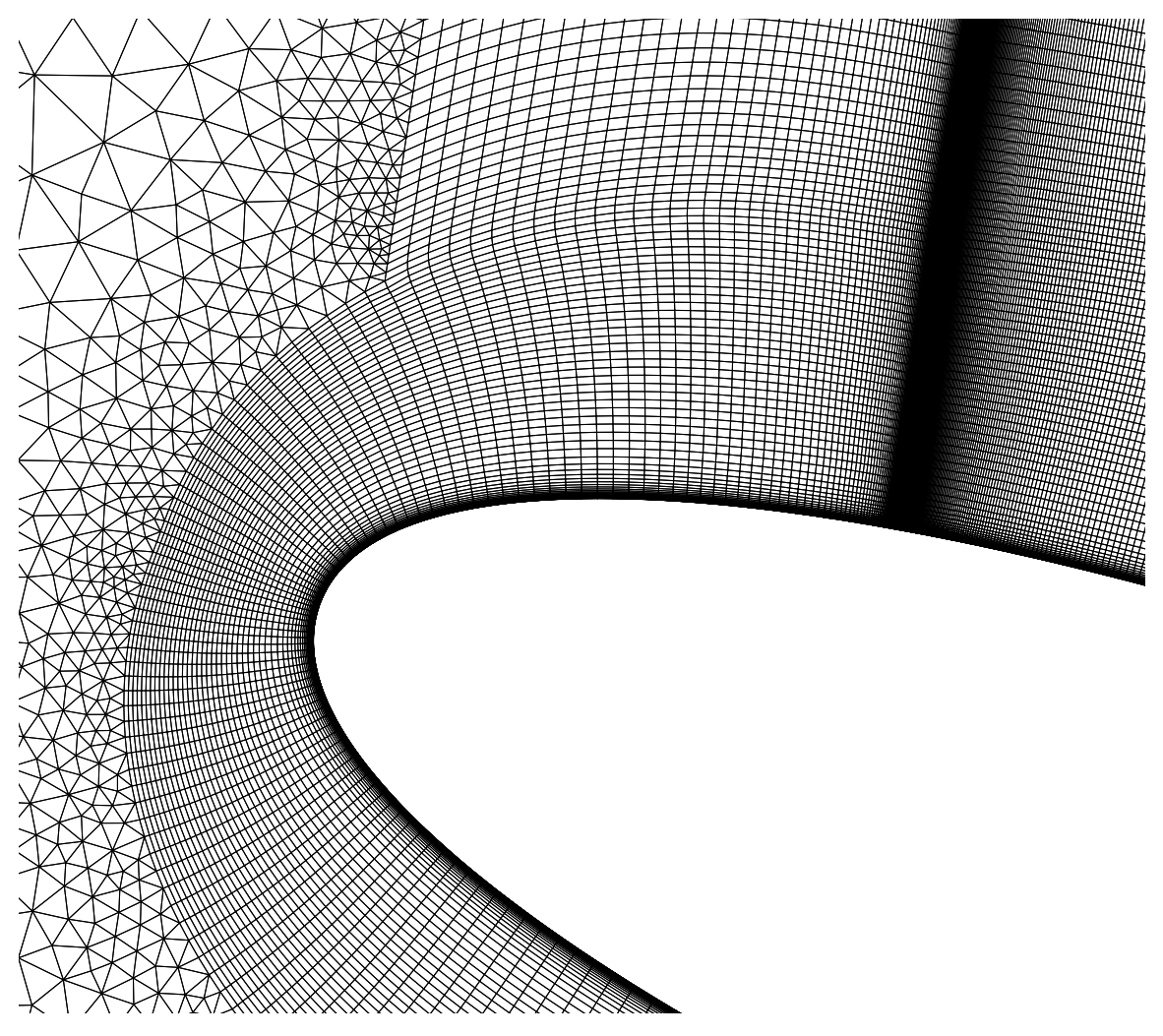

Figure 2: Mesh close to the jet 

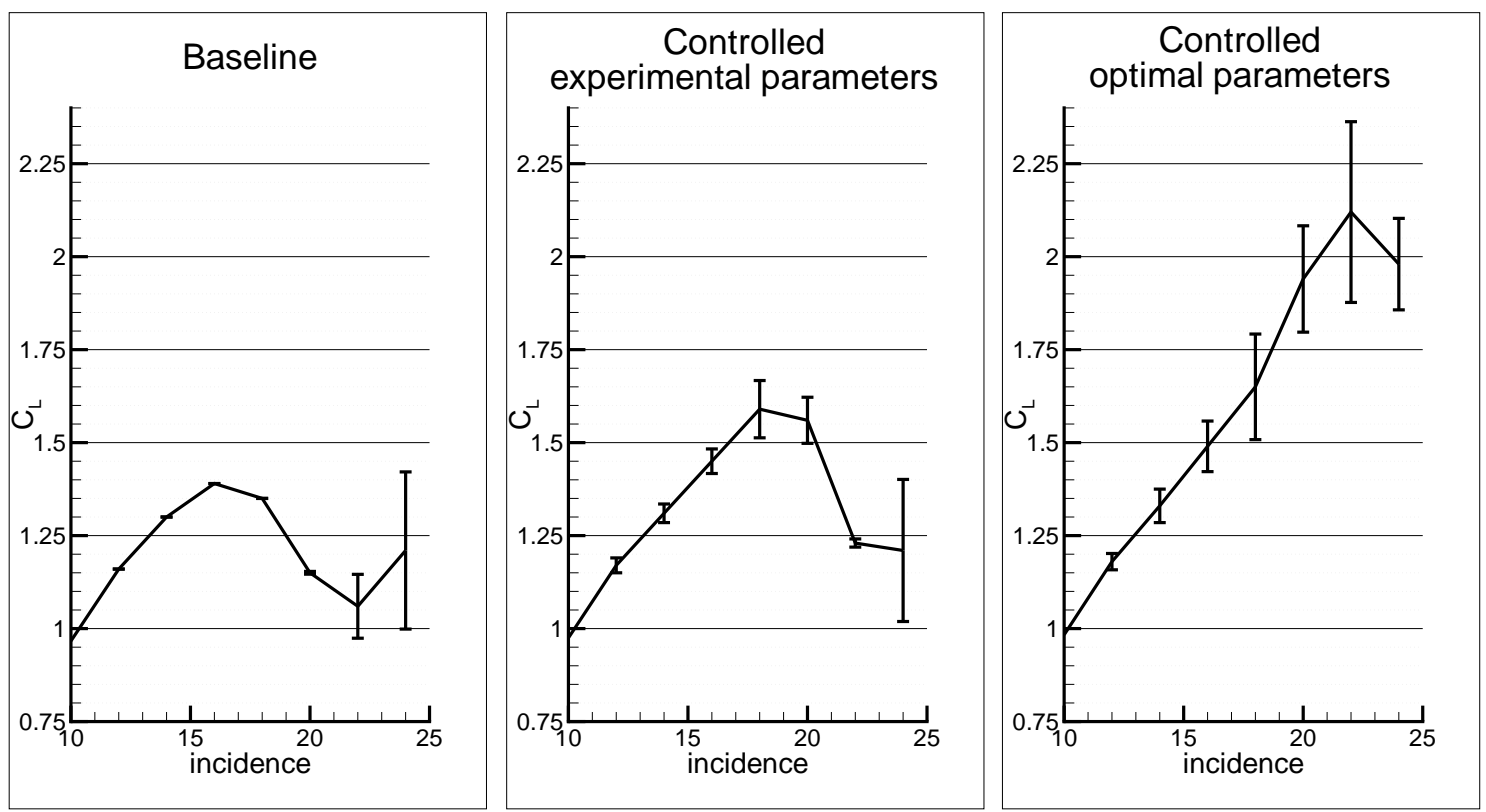

Figure 3: Lift coef. with respect to the angle of attack 

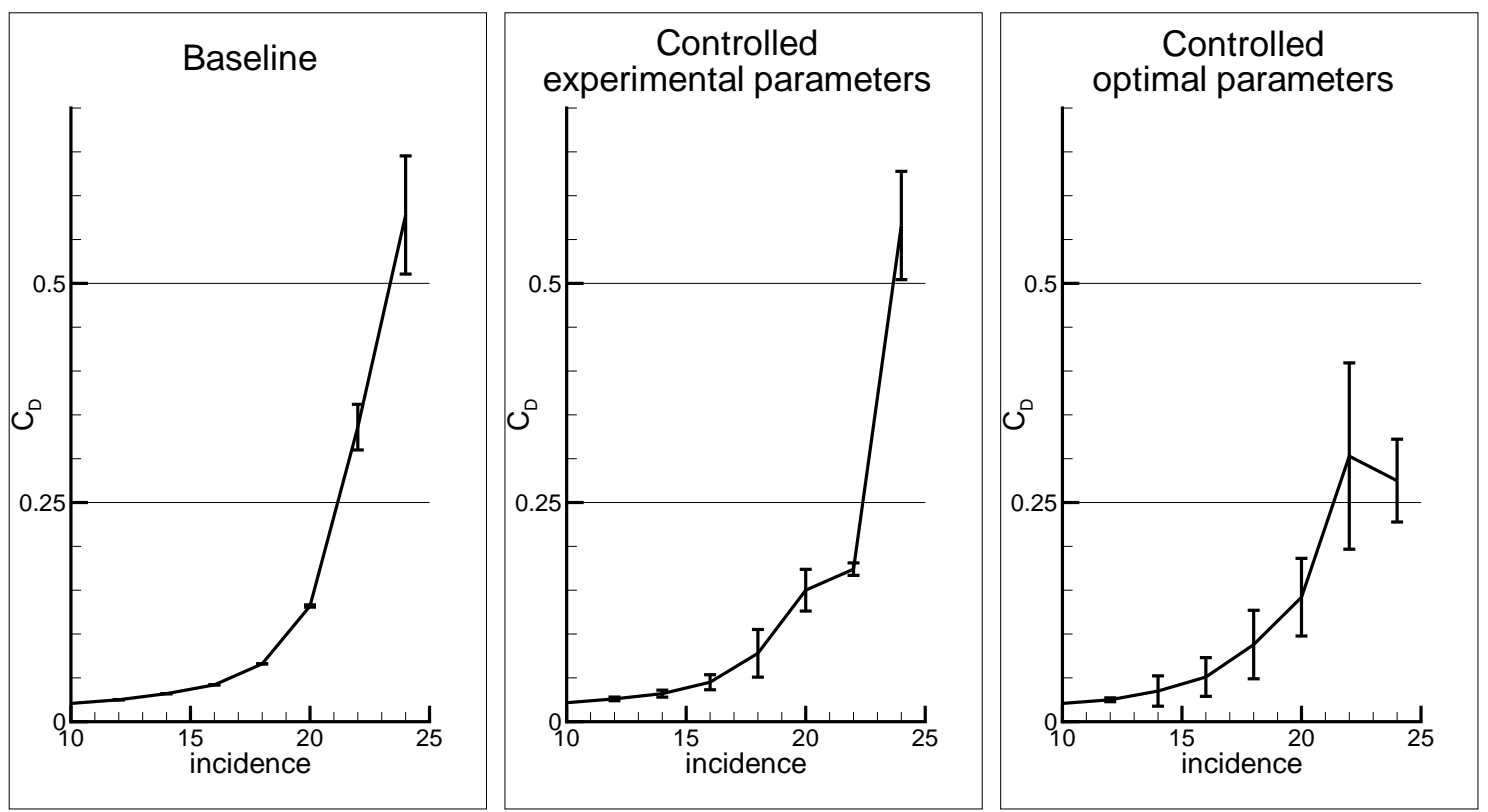

Figure 4: Drag coef. with respect to the angle of attack 


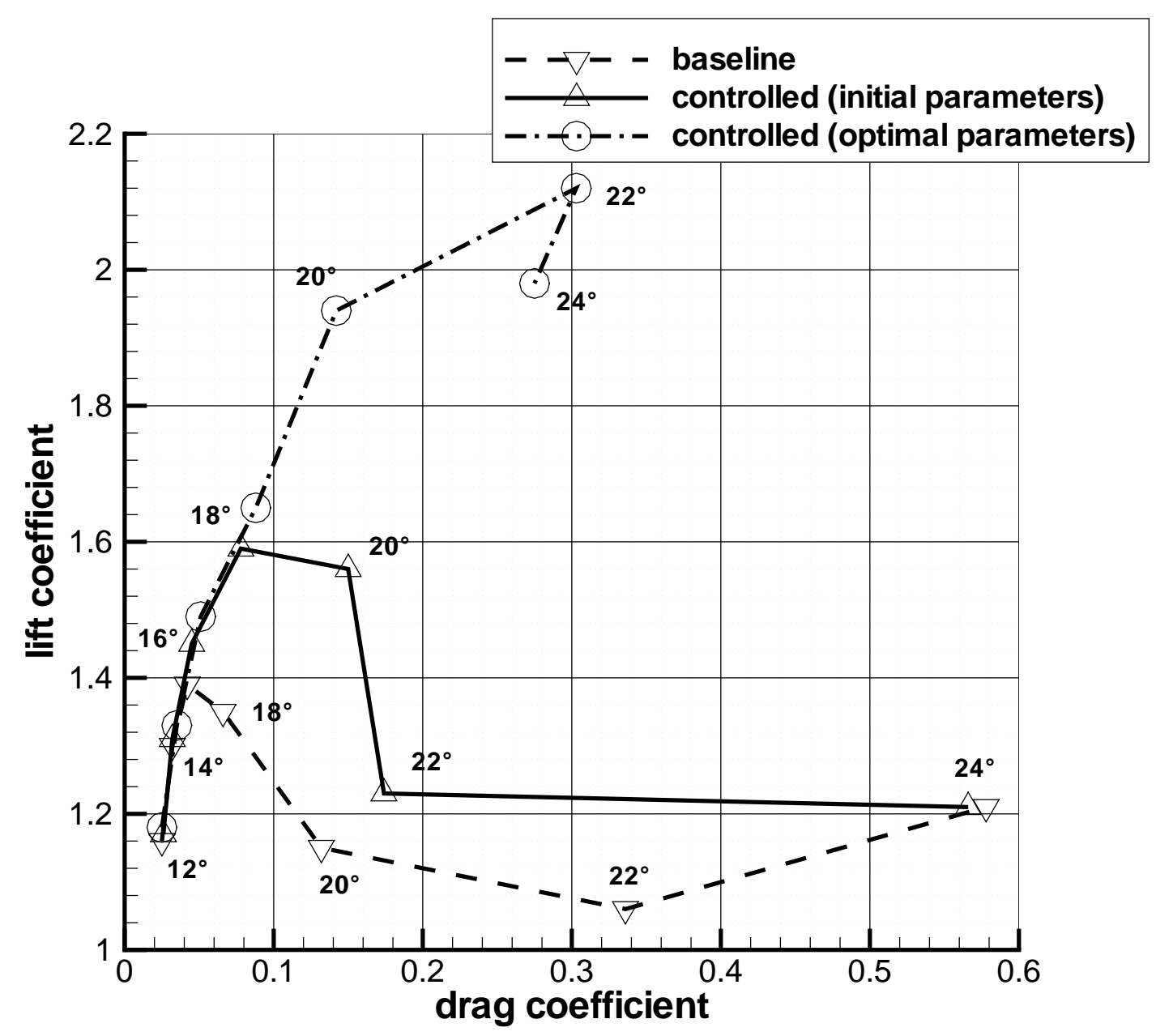

Figure 5: Drag polar 


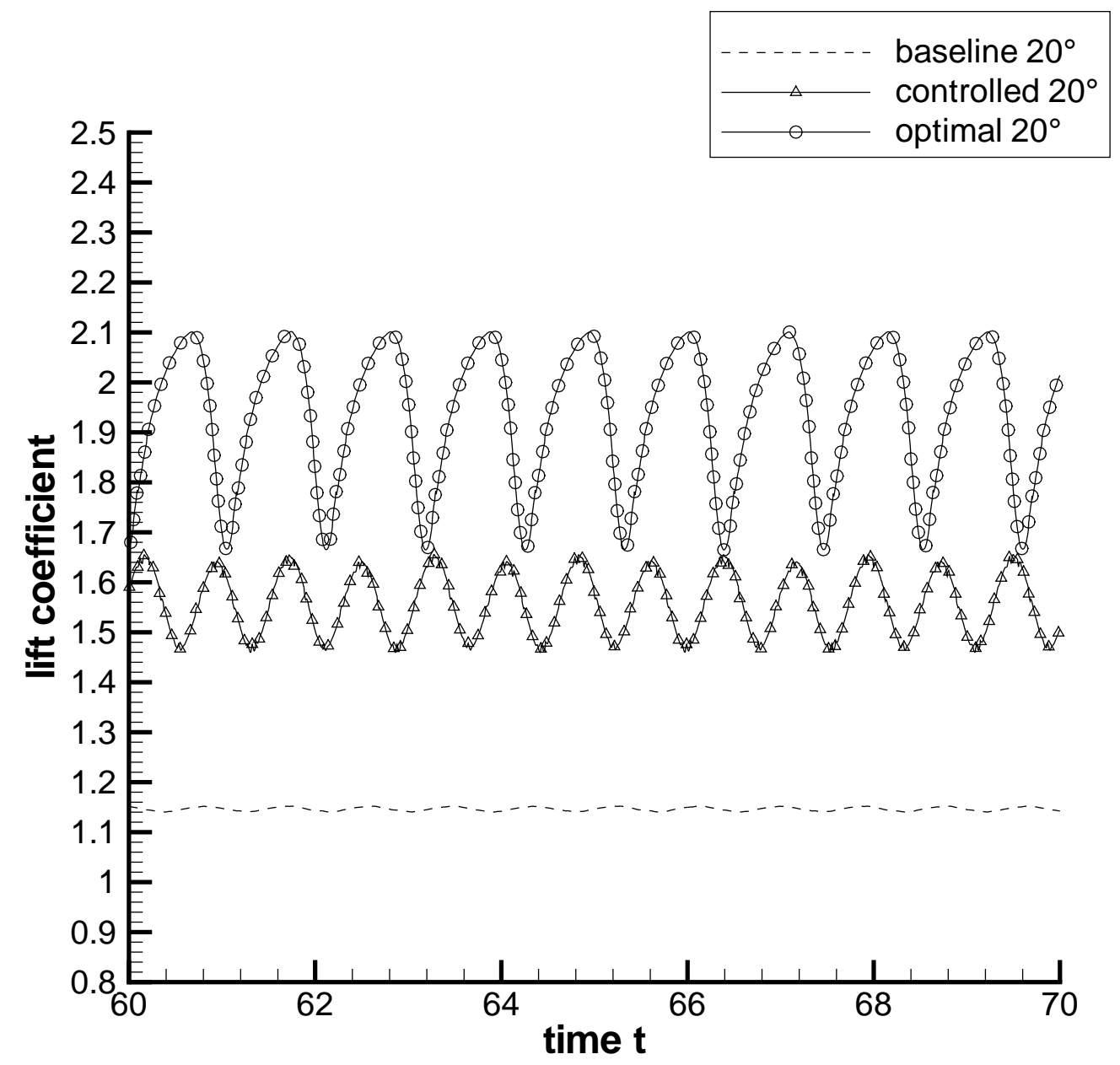

Figure 6: Lift coef. history at $20^{\circ}$ 


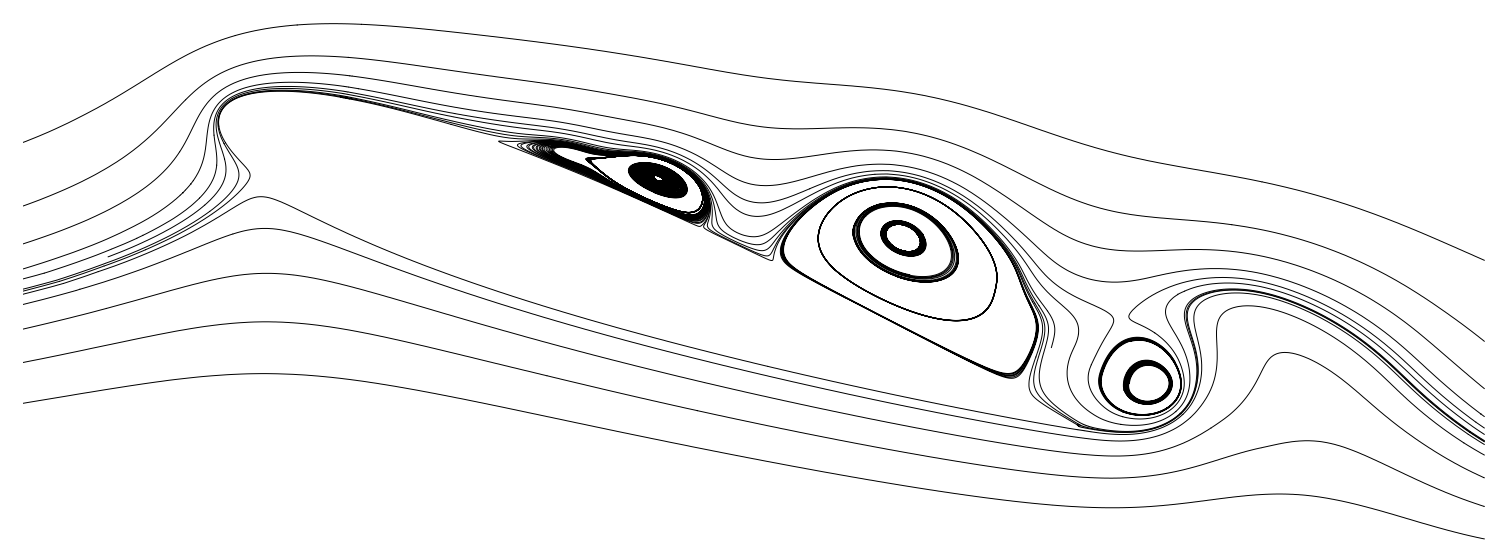

(a) Experimental parameters

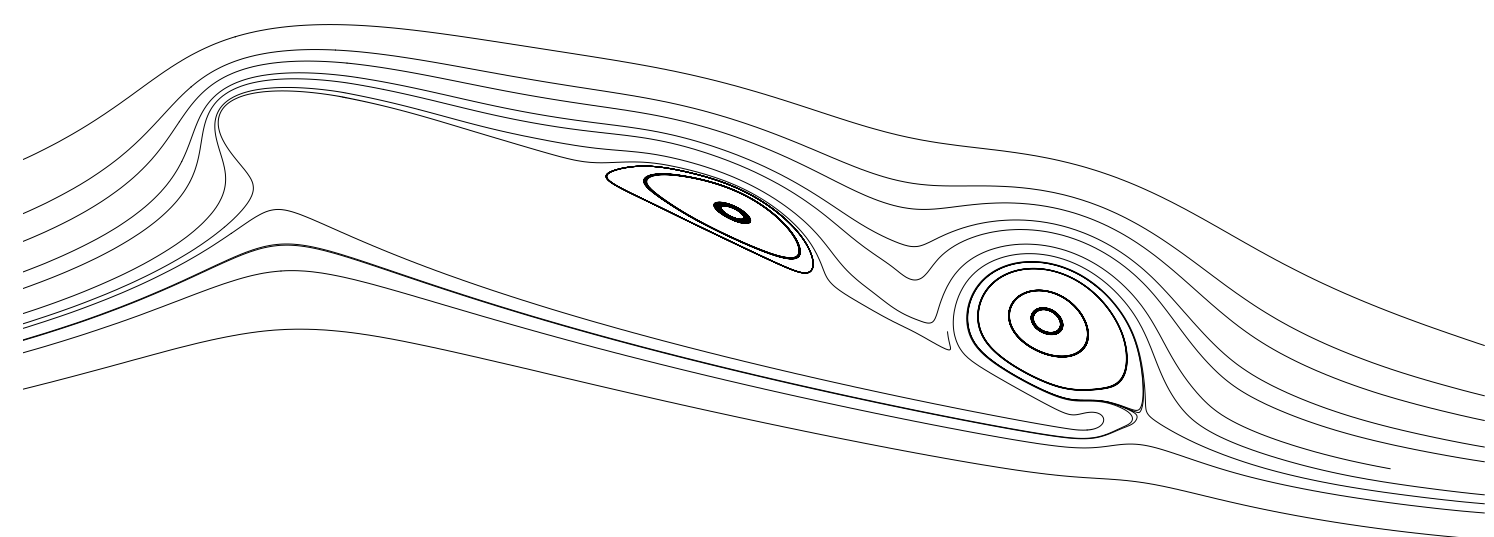

(b) Optimal parameters

Figure 7: Comparison of the streamlines for $\Phi=0$ 


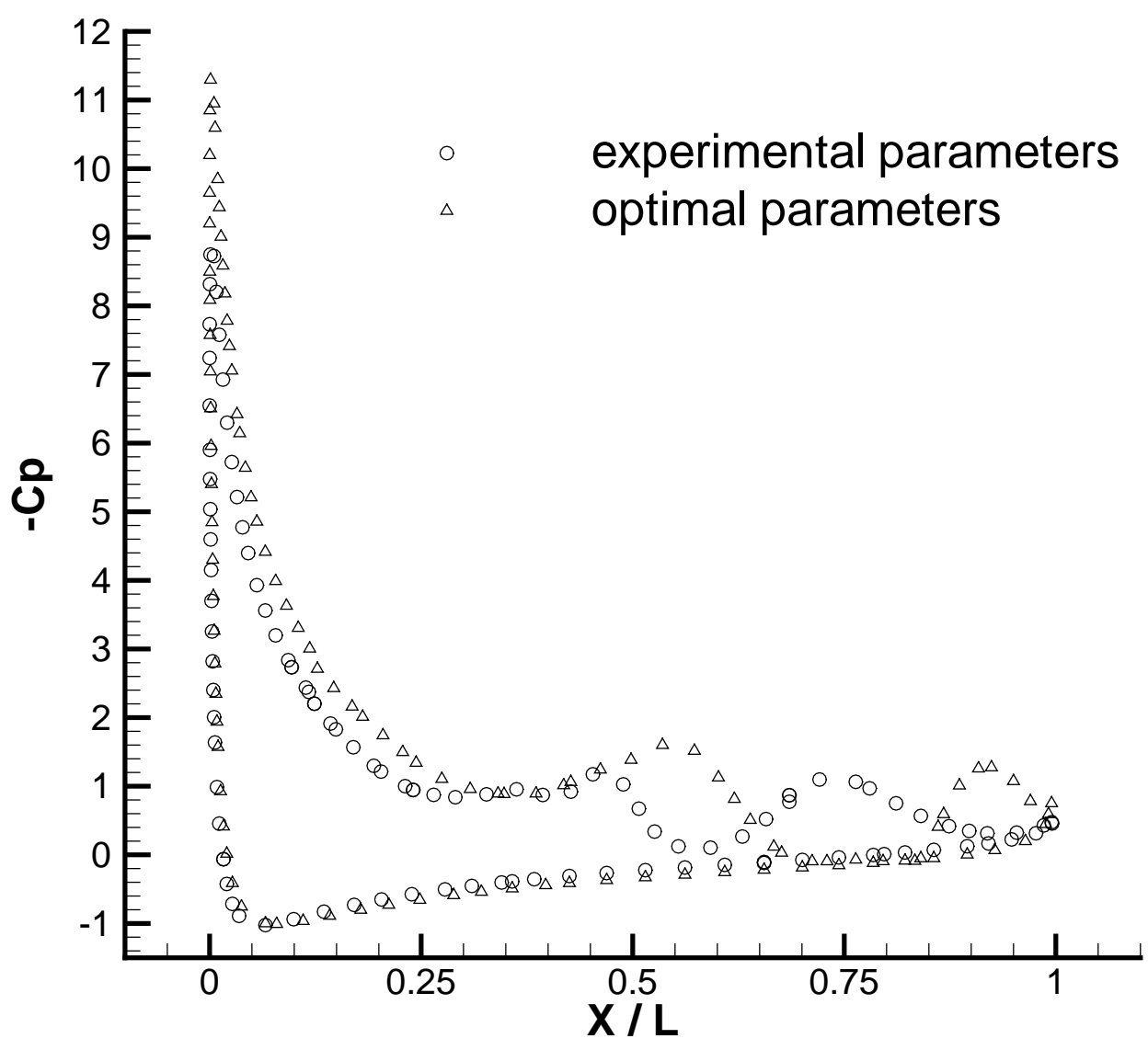

Figure 8: Comparison of the pressure coefficients for $\Phi=0$, computed for experimental and optimal parameters 

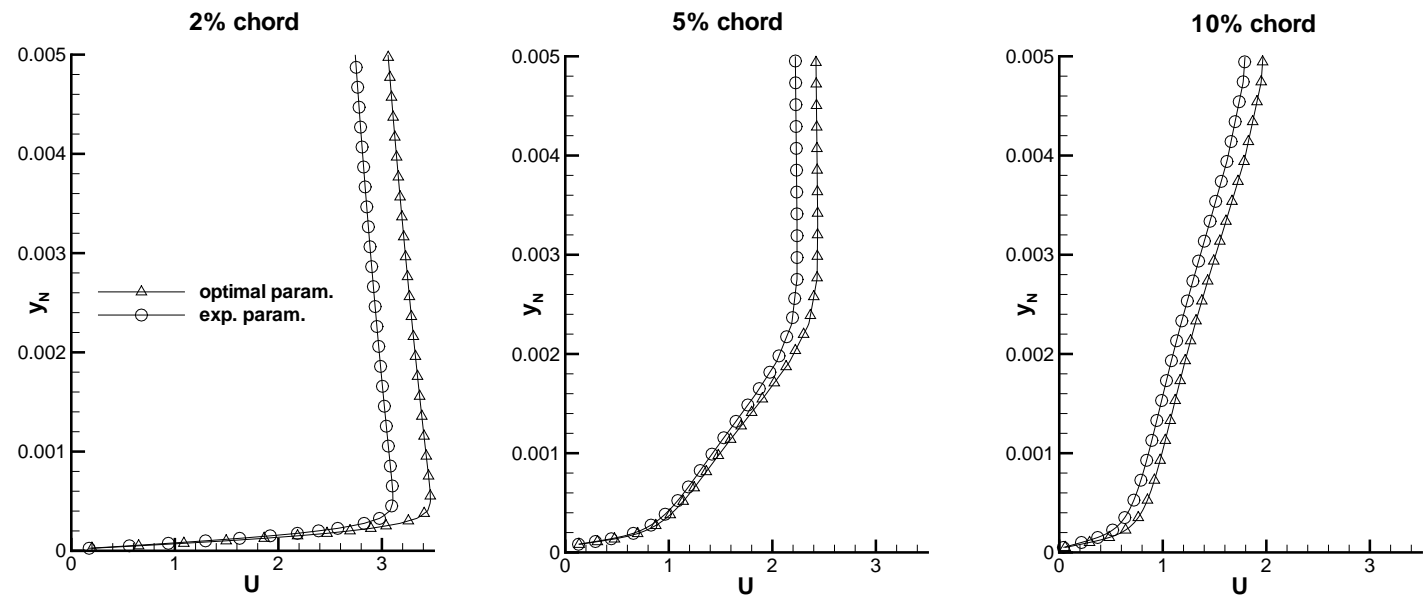

Figure 9: Comparison of the velocity profiles for $\Phi=0$, computed for experimental and optimal parameters 

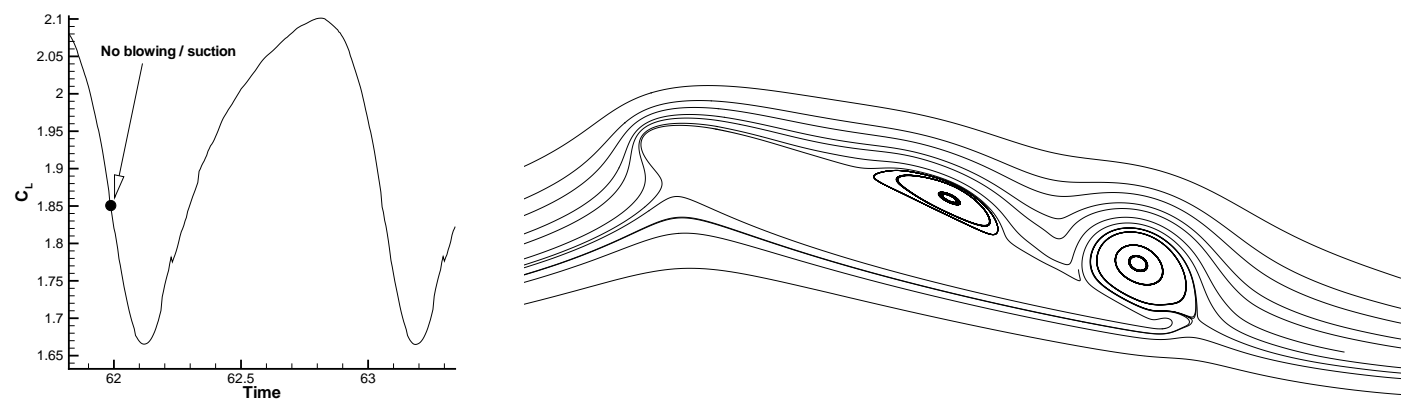

(a) $\Phi=0$
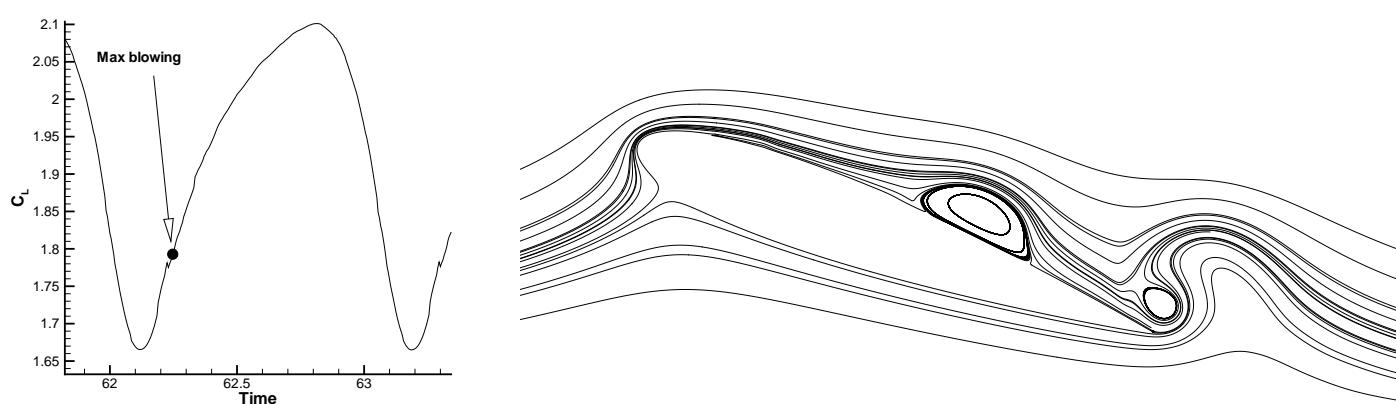

(b) $\Phi=\pi / 2$
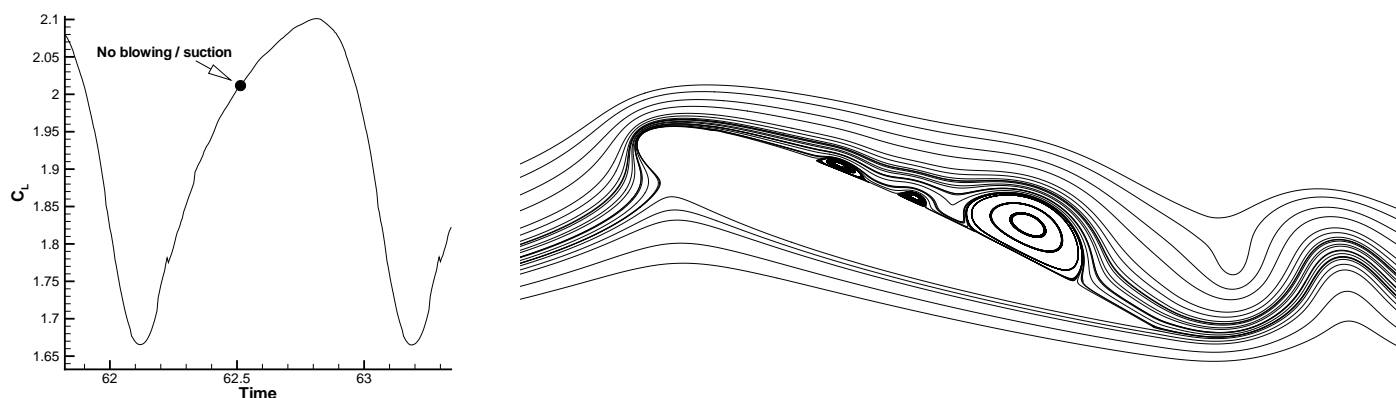

(c) $\Phi=\pi$
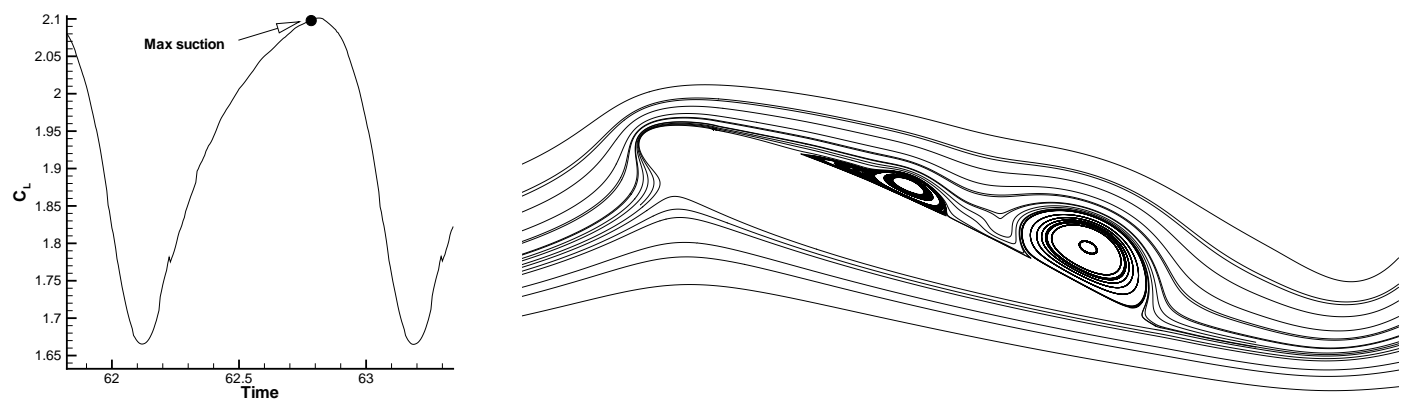

(d) $\Phi=3 \pi / 2$

Figure 10: Streamlines for different times 


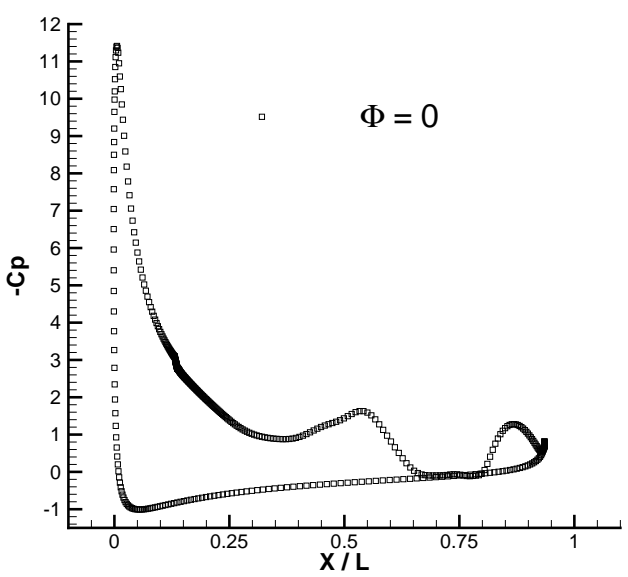

(a) $\Phi=0$

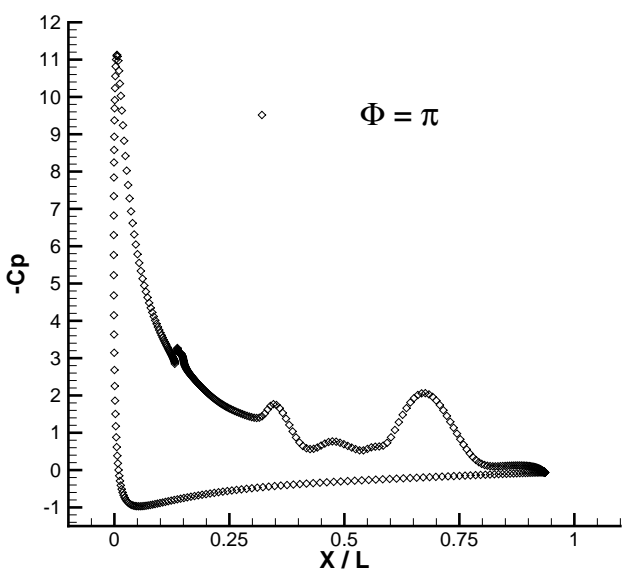

(c) $\Phi=\pi$

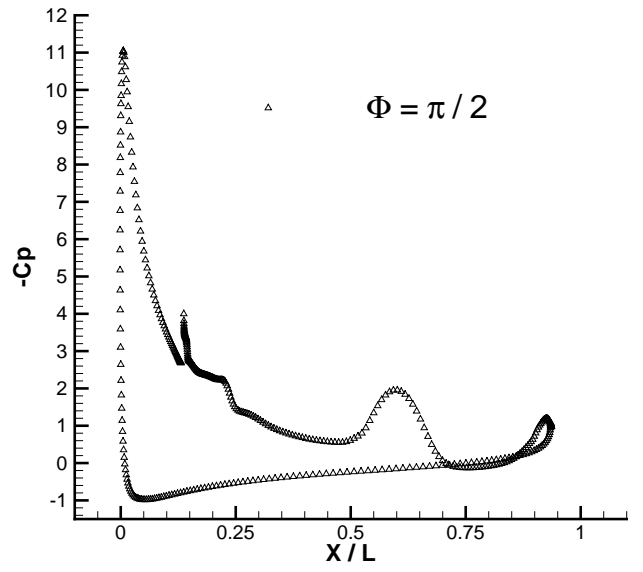

(b) $\Phi=\pi / 2$

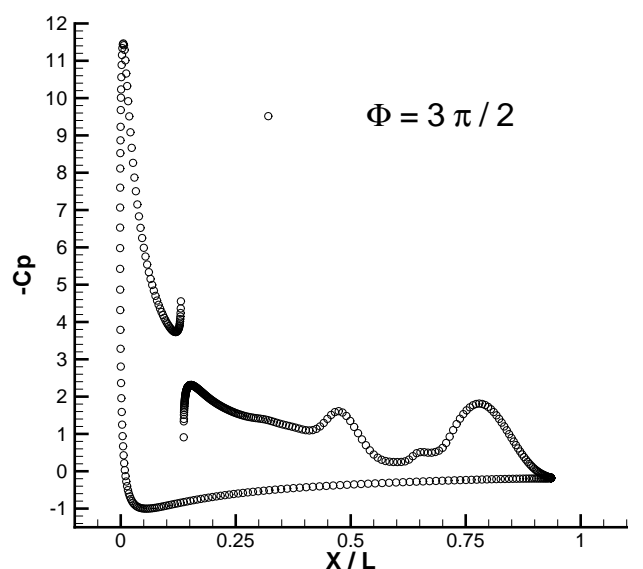

(d) $\Phi=3 \pi / 2$

Figure 11: Pressure coefficients for different times 

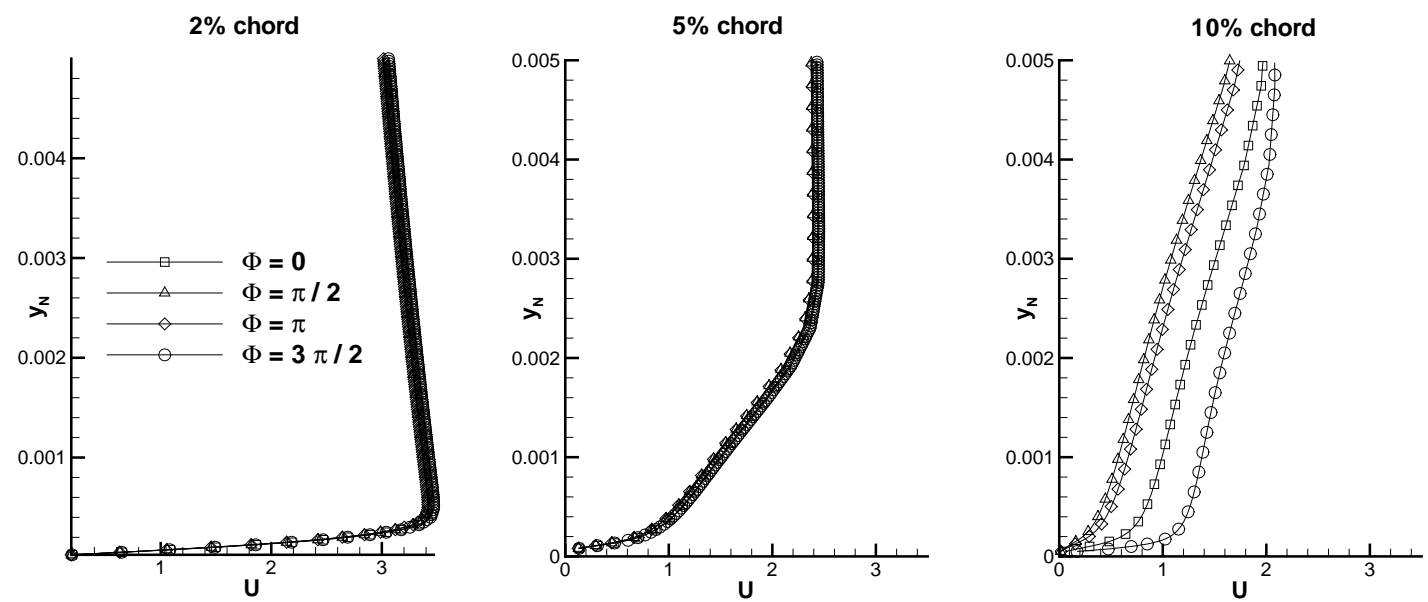

Figure 12: Velocity profiles for optimal parameters 


\section{List of Tables}

1 Optimal parameters found at different angles of attack 


\begin{tabular}{|c|c|c|c|}
\hline & $U_{\text {jet }}$ & $N_{\text {jet }}$ & $\alpha_{\text {jet }}$ \\
\hline Exp. & 1.37 & 1.29 & 10.00 \\
\hline Opt. $12^{\circ}$ & 1.94 & 1.28 & 10.00 \\
\hline Opt. $14^{\circ}$ & 1.76 & 0.88 & 30.15 \\
\hline Opt. $16^{\circ}$ & 1.36 & 0.88 & 27.51 \\
\hline Opt. $18^{\circ}$ & 1.76 & 0.88 & 31.25 \\
\hline Opt. $20^{\circ}$ & 1.61 & 0.93 & 19.68 \\
\hline Opt. $22^{\circ}$ & 1.87 & 0.25 & 35.00 \\
\hline Opt. $24^{\circ}$ & 1.74 & 0.81 & 25.95 \\
\hline
\end{tabular}

Table 1: Optimal parameters found at different angles of attack 